\title{
ON THE MØLLER ENERGY ASSOCIATED WITH BLACK HOLES
}

\author{
Mustafa Saltı and Oktay Aydogdu \\ Department of Physics, Faculty of Art and Science, \\ Middle East Technical University, 06531, Ankara-Turkey \\ E-mail(s): musts6@yahoo.com,oktay231@yahoo.com
}

\begin{abstract}
In this paper, we consider both Einstein's theory of general relativity and the teleparallel gravity (the tetrad theory of gravitation) analogs of the energy-momentum definition of Møller in order to explicitly evaluate the energy distribution (due to matter and fields including gravity) associated with a general black hole model which includes several well-known black holes. To calculate the special cases of energy distribution, here we consider eight different types of black hole models such as anti-de Sitter Cmetric with spherical topology, charged regular black hole, conformal scalar dyon black hole, dyadosphere of a charged black hole, regular black hole, charged topological black hole, charged massless black hole with a scalar field, and the Schwarzschild-de Sitter space-time. Our teleparallel gravitational result is also independent of the teleparallel dimensionless coupling constant, which means that it is valid not only in teleparallel equivalent of general relativity but also in any teleparallel model. This paper also sustains (a) the importance of the energy-momentum definitions in the evaluation of the energy distribution of a given spacetime and (b) the viewpoint of Lessner that the Møller energy-momentum complex is the powerful concept to calculate energy distribution in a given space-time.
\end{abstract}

Keywords: Møller energy; black holes; general relativity; teleparallel gravity.

\section{INTRODUCTION}

It is possible to evaluate the energy distribution and momentum by using various energymomentum complexes. A large number of formulations of the gravitational energy, momentum and angular momentum have been given. Some of them are coordinate independent and other are coordinate-dependent. There lies a dispute on the importance of non-tensorial energy-momentum complexes whose physical interpretations have been questioned by a number of physicists, including Weyl, Pauli and Eddington. Also, 
there exists an opinion that the energy-momentum pseudo-tensors are not useful to find meaningful results in a given geometry. Ever since the Einstein's energy-momentum complex [1], used for calculating energy and momentum in a general relativistic system, many attempts have been made to evaluate the energy distribution for a given spacetime [2]. Except for the one which was defined by Møller, these definitions only give meaningful results if the calculations are performed in "Cartesian" coordinates. Møller constructed an expression which enables one to evaluate energy and momentum in any coordinate system. Lessner [3] argued that the Møller prescription is a powerful concept of energy-momentum in general relativity.

Several examples of particular space-times have been investigated and different energy-momentum formulations are known to give the same energy distribution for a given space-time [4]. For instance, in Phys. Rev. D60-104041 (1999), Virbhadra, using the energy and momentum complexes of Einstein, Landau-Lifshitz, Papapetrou and Weinberg for a general non-static spherically symmetric metric of the Kerr-Schild class, showed that all of these energy-momentum formulations give the same energy distribution as in the Penrose energy-momentum formulation. Albrow [5] and Tryon [6] assumed that the net energy of the universe may be equal to zero. The subject of the energy-momentum distributions of closed and open universes was initiated by an interesting work of Cooperstock and Israelit [7]. They found the zero value of energy for any homogenous isotropic universe described by a Friedmann-Robertson-Walker metric in the context of general relativity. This interesting result influenced some general relativists [8].

Recently, the problem of energy-momentum localization has also been considered in teleparallel gravity [9]. The authors found that energy-momentum also localize in this alternative theory of gravitation, and their results agree with some previous papers which were studied in the general theory of relativity. In Gen. Relat. Gravit. 36, 1255(2004); Vargas, using the definitions of Einstein and Landau-Lifshitz in teleparallel gravity, found that the total energy is zero in Friedmann-Robertson-Walker spacetimes. Recently, Saltı, Aydogdu and their collaborators [10] have calculated the energymomentum density using different complexes for a given space-time both in general relativity and the teleparallel theory of gravity and obtained the same results.

The paper is organized as follows. In the next section, we introduce a general black model, and give some well-known black hole solutions as examples for the general model. Next, in section 3, we give the energy-momentum definitions of Møller both in Einstein's theory of general relativity and the teleparallel gravity. Section 4 gives us the calculations for the energy distribution. In section 4, we compute the energy of seven special black hole models introduced in section 1. Finally, section 5 is devoted to summary and conclusions.

Notations and conventions: $c=h=1$, metric signature $(-,+,+,+)$, Greek indices run from 0 to 3 and, Latin ones from 1 to 3. Throughout this paper, Latin indices $(i, j$, ...) number the vectors, and Greek indices $(\mu, \nu, \ldots)$ represent the vector components. 
On the Møller energy associated with black holes

\section{Black hole models to be considered}

A general black hole model can be given as

$$
g_{\mu \nu} d x^{\mu} d x^{\nu}=V(r) d t^{2}-V(r)^{-1} d r^{2}-r^{2}\left[d \theta^{2}-\sin ^{2} \theta d \phi^{2}\right] .
$$

This space-time model can be reduced to some well-known black holes under special choices of $V(r)$. One can easily find lots of models which are special cases of our general line-element. Here we give a few of them as examples.

1. Anti-de Sitter C-metric with spherical topology; This black hole model can be obtained by choosing [11, 12 ]

$$
V(r)=1-\frac{2 m}{r}+\frac{q^{2}}{r^{2}}+\frac{|\Lambda|}{3} r^{2} .
$$

This model has a cosmological horizon and its charged version has only one black hole horizon (as opposed to the charged case which has an inner and an outer black hole horizon).

2. Charged regular black hole; The Reissner-Nordstrom [13] solution represents an electrically charged black hole. This is also special case of the anti-de Sitter C-metric, and it can be defined by choosing $\Lambda=0$.

$$
V(r)=1-\frac{2 m}{r}+\frac{q^{2}}{r^{2}}
$$

where $q$ and $m$ are the electric charge and respectively the mass of the black hole.

A solution to the coupled system of the Einstein field and equations of the nonlinear electrodynamics was recently given by E. Ayon-Beato and A. Garcia [14. This solution represents a regular black hole with mass $m$ and electric charge $q$ and avoids thus the singularity problem. Also, the metric at large distances behaves as the ReissnerNordstrom solution. The usual singularity of the Reissner-Nordstrom solution, at $r=0$, has been smoothed out and now it simply corresponds to the origin of the spherical coordinates. The line-element is given by (11) with

$$
V(r)=1-\frac{2 m}{r}\left(1-\tanh \left(\frac{q^{2}}{2 m r}\right)\right) .
$$

If the electric charge vanishes, we reach the Schwarzschild solution. At large distance (44) resembles the Reissner-Nordstrom solution and can be written as

$$
V(r)=1-\frac{2 m}{r}+\frac{q^{2}}{r^{2}}-\frac{q^{6}}{12 m^{2} r^{6}}+O\left(\frac{1}{r^{6}}\right) .
$$

3. Conformal scalar dyon black hole; Virbhadra [15] gave an exact solution of Einstein-Maxwell conformal scalar field equations which is a black hole solution and is characterized by scalar, charge, magnetic charge and electric charge. This solution is given by the line element which is defined by the conditions

$$
V(r)=\left(1-\frac{Q_{C S D}}{r}\right)^{2}
$$


and the conformal scalar field is given by

$$
\psi=\sqrt{\frac{3}{4 \pi}}\left(\frac{q_{s}}{r-Q_{C S D}}\right)
$$

with $q_{s}$ the scalar charge and where

$$
Q_{C S D}=\sqrt{q_{s}^{2}+q_{e}^{2}+q_{m}^{2}}
$$

so, this case describes A Conformal Scalar Dyon Black Hole solutions.

4. Dyadosphere of a charged black hole; The event horizon of a charged black hole is, according to Ruffini [16] and Preparata et. al. [17], surrounded by a special region called the dyadosphere where the electromagnetic field exceeds the Euler-Heisenberg critical value for electron-positron pair production. The new concept of dyadosphere of an electromagnetic black hole was introduced by Ruffini to explain gamma ray bursts. Ruffini defined the dyadosphere as the region just outside the horizon of a charged black hole whose electromagnetic field strength is larger than the well-known Heisenberg-Euler critical value

$$
\Xi_{\text {critical }}=\frac{m_{e}^{2} c^{3}}{r \hbar}
$$

where $m_{e}$ and $e$ denote mass and charge of an electron, respectively. For a ReissnerNordstrom black hole, the dyadosphere is defined by the radial interval $r_{+} \leq r \leq r_{d s}$ where the horizon

$$
r_{+}=\frac{G M}{c^{2}}\left(1+\sqrt{1-\frac{q^{2}}{G M^{2}}}\right)
$$

forms the inner radius of the dyadosphere, while its outer radius is given by

$$
r_{d s}=\sqrt{\frac{\hbar}{c m_{e}} \frac{G M}{c^{2}} \frac{m_{p}}{m_{e}} \frac{e}{q_{p}} \frac{Q}{M \sqrt{G}}}
$$

where $M, Q, m_{p}=\sqrt{\frac{\hbar c}{G}}$ and $q_{c}=\sqrt{\hbar c}$ are mass, charge parameters, the Planck mass and the Planck charge, respectively. The total energy of electron-positron pairs converted from static electric energy and deposited within the dydosphere is obtained [18] as

$$
E_{d y a}=\frac{Q^{2}}{2 r_{+}}\left(1-\frac{r_{-}}{r_{d s}}\right)\left(1-\frac{r_{+}^{2}}{r_{d s}^{2}}\right) .
$$

De Lorenci, Figueiredo, Fliche and Novello [19] found the correction for the Reissner-Nordstrom metric from the first contribution of the Euler-Heisenberg Lagrangian and obtained the following condition for the line-element (1)

$$
V(r)=1-\frac{2 M}{r}+\frac{Q^{2}}{r^{2}}-\frac{\sigma Q^{4}}{5 r^{6}} .
$$

By writing $\sigma=0$, we obtain the Reissner-Nordstrom space-time. De Lorenci et. al. showed that the correction term $\frac{\sigma Q^{4}}{5 R^{6}}$ is of the same order of magnitude as the ReissnerNordstrom charge term $\frac{Q^{2}}{2 R^{2}}$.

5. Regular black hole; Bardeen [20, 21] constructed a well-known model that represents a regular black hole obeying the weak energy condition. And it was powerful 
in shaping the direction of research on the existence or avoidance of singularities. This model uses the Reissner-Nordstrom metric as inspiration. The metric expressed in standard spherical coordinates $(t, r, \theta, \phi)$ is given by the condition

$$
V^{2}(r)=1-\frac{2 m r^{2}}{\left(r^{2}+e^{2}\right)^{3 / 2}}
$$

when $e^{2}<\frac{16}{27} m^{2}$, there is an event horizon. There are values $R_{ \pm}$of $R$ such that the region $R_{-}<R<R_{+}$contains trapped surfaces. The model obeys the null convergence, yet it contains no physical singularities. It is to be noticed that if we take charge $e=0$, the case given above reduces to the Schwarzschild metric.

6. Charged topological black hole; The line element [22] can be given with

$$
V(r)=-\frac{\Lambda r^{2}}{3}-\left(1+\frac{G m}{r^{2}}\right)^{2}
$$

where $-\infty<t<\infty$ and $r>0$. The scalar field is

$$
\phi=\sqrt{\frac{-\Lambda}{6 \alpha}} \frac{G \mu}{r+G \mu}
$$

with $\alpha>0$ (the scalar field is real provided $\alpha>0$ ) and the only non-zero component of electromagnetic field is

$$
A_{t}=-q r^{-1} \text {. }
$$

The integration constants $q$ and $\mu$ are not independent. They are related as

$$
q^{2}=-G \mu^{2}\left(1+\frac{2 \pi \Lambda G}{9 \alpha}\right) .
$$

The mass $M$, and the electric charge $Q$ are given by

$$
M=\frac{\sigma}{4 \pi} \mu, \quad Q=\frac{\sigma}{4 \pi} q
$$

respectively, where $\sigma$ denotes the area of $\Sigma$.

7. Charged massless black hole with a scalar field; This model can be obtained from the charged topological black hole solution by taking $m=0$ [22],

$$
V(r)=-\frac{\Lambda r^{2}}{3}-1
$$

8. The Schwarzschild-de Sitter space-time;

$$
V(r)=1-\frac{2 M}{r}-\frac{r^{2}}{l^{2}}
$$

here $M$ is the mass of the black hole, and $l^{2}$ is related to the positive cosmological constant. The space-time model has more than one horizon if $0<\chi<\frac{1}{27}$ where $\chi=\frac{M^{2}}{l^{2}}$.

The black hole horizon $r_{h}$ and the cosmological horizon $r_{c}$ are located [23], respectively, at

$$
r_{h}=\frac{2 M}{\sqrt{3 \chi}} \cos \frac{\pi+\Xi}{3}
$$


On the Møller energy associated with black holes

$$
r_{c}=\frac{2 M}{\sqrt{3 \chi}} \cos \frac{\pi-\Xi}{3}
$$

where

$$
\Xi=\arccos (3 \sqrt{3 \chi})
$$

\section{The Energy Formulation of Møller}

\subsection{In General Relativity}

The energy and/or momentum complex of Møller [2] in general relativity is given by

$$
M_{\mu}^{\nu}=\frac{1}{8 \pi} \chi_{\mu, \alpha}^{\nu \alpha}
$$

satisfying the local conservation laws:

$$
\frac{\partial M_{\mu}^{\nu}}{\partial x^{\nu}}=0
$$

where the antisymmetric super-potential $\chi_{\mu}^{\nu \alpha}$ is

$$
\chi_{\mu}^{\nu \alpha}=\sqrt{-g}\left[g_{\mu \beta, \gamma}-g_{\mu \gamma, \beta}\right] g^{\nu \gamma} g^{\alpha \beta} .
$$

The locally conserved energy-momentum complex $M_{\mu}^{\nu}$ contains contributions from the matter, non-gravitational and gravitational fields. $M_{0}^{0}$ is the energy density and $M_{a}^{0}$ are the momentum density components. The momentum four-vector of Møller is given by

$$
P_{\mu}=\iiint M_{\mu}^{0} d x d y d z
$$

Using Gauss's theorem, this definition transforms into

$$
P_{\mu}=\frac{1}{8 \pi} \iint \chi_{\mu}^{0 a} \mu_{\alpha} d S
$$

where $\mu_{a}$ (where $a=1,2,3$ ) is the outward unit normal vector over the infinitesimal surface element $d S$. $P_{i}$ give momentum components $P_{1}, P_{2}, P_{3}$ and $P_{0}$ gives the energy.

\subsection{In the Tetrad Theory of Gravitation}

The teleparallel theory of gravity (the tetrad theory of gravitation) is an alternative approach to gravitation and corresponds to a gauge theory for the translation group based on Weitzenböck geometry [24]. In the theory of teleparallel gravity, gravitation is attributed to torsion [25], which plays the role of a force [26], and the curvature tensor vanishes identically. The essential field is acted by a nontrivial tetrad field, which gives rise to the metric as a by-product. The translational gauge potentials appear as the nontrivial item of the tetrad field, so induces on space-time a teleparallel structure which is directly related to the presence of the gravitational field. The interesting place of teleparallel theory is that, due to its gauge structure, it can reveal a more appropriate approach to consider some specific problem. This is the situation, for example, in the energy and momentum problem, which becomes more transparent. 
Møller modified general relativity by constructing a new field theory in teleparallel space. The aim of this theory was to overcome the problem of the energy-momentum complex that appears in Riemannian Space [27]. The field equations in this new theory were derived from a Lagrangian which is not invariant under local tetrad rotation. Saez [28] generalized Møller theory into a scalar tetrad theory of gravitation. Meyer [29] showed that Møller theory is a special case of Poincare gauge theory [30, 31].

In teleparallel gravity, the superpotential of Møller is given by Mikhail et al. [9] as

$$
U_{\mu}^{\nu \beta}=\frac{(-g)^{1 / 2}}{2 \kappa} P_{\chi \rho \sigma}^{\tau \nu \beta}\left[\Phi^{\rho} g^{\sigma \chi} g_{\mu \tau}-\lambda g_{\tau \mu} \xi^{\chi \rho \sigma}-(1-2 \lambda) g_{\tau \mu} \xi^{\sigma \rho \chi}\right]
$$

where $\xi_{\alpha \beta \mu}=h_{i \alpha} h_{\beta ; \mu}^{i}$ is the con-torsion tensor and $h_{i}{ }^{\mu}$ is the tetrad field and defined uniquely by $g^{\alpha \beta}=h_{i}^{\alpha} h_{j}^{\beta} \eta^{i j}$ (here $\eta^{i j}$ is the Minkowski space-time). $\kappa$ is the Einstein constant and $\lambda$ is free-dimensionless coupling parameter of teleparallel gravity. For the teleparallel equivalent of general relativity, there is a specific choice of this constant.

$\Phi_{\rho}$ is the basic vector field given by

$$
\Phi_{\mu}=\xi^{\rho}{ }_{\mu \rho}
$$

and $P_{\chi \rho \sigma}^{\tau \nu \beta}$ can be found by

$$
P_{\chi \rho \sigma}^{\tau \nu \beta}=\delta_{\chi}^{\tau} g_{\rho \sigma}^{\nu \beta}+\delta_{\rho}^{\tau} g_{\sigma \chi}^{\nu \beta}-\delta_{\sigma}^{\tau} g_{\chi \rho}^{\nu \beta}
$$

with $g_{\rho \sigma}^{\nu \beta}$ being a tensor defined by

$$
g_{\rho \sigma}^{\nu \beta}=\delta_{\rho}^{\nu} \delta_{\sigma}^{\beta}-\delta_{\sigma}^{\nu} \delta_{\rho}^{\beta} .
$$

The energy-momentum density is defined by

$$
\Sigma_{\alpha}^{\beta}=U_{\alpha, \lambda}^{\beta \lambda}
$$

where comma denotes ordinary differentiation. The energy is expressed by the surface integral;

$$
E=\lim _{r \rightarrow \infty} \int_{r=\text { constant }} U_{0}^{0 \zeta} \eta_{\zeta} d S
$$

where $\eta_{\zeta}$ is the unit three-vector normal to surface element $d S$.

\section{Calculations}

In this part of the study, we will calculate the energy distribution (due to matter and fields including gravity) for a general metric that includes seven black hole models introduced in section 1.

\subsection{In Genaral Relativity}

The matrix of the $g_{\mu \nu}$ is defined by

$$
\left(\begin{array}{cccc}
V(r) & 0 & 0 & 0 \\
0 & -\frac{1}{V(r)} & 0 & 0 \\
0 & 0 & -r^{2} & 0 \\
0 & 0 & 0 & -r^{2} \sin ^{2} \theta
\end{array}\right)
$$


On the Møller energy associated with black holes

and its inverse $g^{\mu \nu}$ is

$$
\left(\begin{array}{cccc}
\frac{1}{V(r)} & 0 & 0 & 0 \\
0 & -V(r) & 0 & 0 \\
0 & 0 & -\frac{1}{r^{2}} & 0 \\
0 & 0 & 0 & -\frac{1}{r^{2} \sin ^{2} \theta}
\end{array}\right) .
$$

For the line-element (11), under consideration, we calculate

$$
\chi_{0}^{01}=\frac{V_{r} r^{2} \sin \theta}{2 \sqrt{V}}
$$

which is the only required component of $\chi_{\mu}^{\nu \alpha}$ for our purpose. Here, the index $r$ indicates derivative with respect to $r$.

\subsection{In Teleparallel Gravity}

The general form of the tetrad, $h_{i}^{\mu}$, having spherical symmetry was given by Robertson [32. In the Cartesian form it can be written as

$$
h_{0}^{0}=i A, \quad h_{a}^{0}=C x^{a}, \quad h_{0}^{\alpha}=i D x^{\alpha}, \quad h_{a}^{\alpha}=B \delta_{a}^{\alpha}+E x^{a} x^{\alpha}+\epsilon_{a \alpha \beta} F x^{\beta}
$$

where $A, B, C, D, E$, and $F$ are functions of $t$ and $r=\sqrt{x^{a} x^{a}}$, and the zeroth vector $h_{0}^{\mu}$ has the factor $i^{2}=-1$ to preserve Lorentz signature and the tetrad of Minkowski space-time is $h_{a}^{\mu}=\operatorname{diag}\left(i, \delta_{a}^{\alpha}\right)$ where $(\mathrm{a}=1,2,3)$.

Using the general coordinate transformation

$$
h_{a \mu}=\frac{\partial \mathbf{X}^{\nu^{\prime}}}{\partial \mathbf{X}^{\mu}} h_{a \nu}
$$

where $\left\{\mathbf{X}^{\mu}\right\}$ and $\left\{\mathbf{X}^{\nu^{\prime}}\right\}$ are, respectively, the isotropic and Schwarzschild coordinates $(t, r, \theta, \phi)$. In the spherical, static and isotropic coordinate system $\mathbf{X}^{1}=r \sin \theta \cos \phi$, $\mathbf{X}^{2}=r \sin \theta \sin \phi, \mathbf{X}^{3}=r \cos \theta$. We obtain the tetrad components as

$$
\left(\begin{array}{cccc}
\frac{i}{\sqrt{V(r)}} & 0 & 0 & 0 \\
0 & \sqrt{V(r)} \sin \theta \cos \phi & \frac{1}{r} \cos \theta \cos \phi & -\frac{\sin \phi}{r \sin \theta} \\
0 & \sqrt{V(r)} \sin \theta \sin \phi & \frac{1}{r} \cos \theta \sin \phi & \frac{\cos \phi}{r \sin \theta} \\
0 & \sqrt{V(r)} \cos \theta & -\frac{1}{r} \sin \theta & 0
\end{array}\right)
$$

where $i^{2}=-1$. Hence, the required non-vanishing component of $U_{\mu}^{\nu \beta}$ is [33, 34]

$$
U_{0}^{01}=\frac{V_{r} r^{2} \sin \theta}{2 \kappa \sqrt{V}}
$$

\section{Solutions for Special Black Hole Models}

In this section, we consider equations (38) and (42) with equations (29) and (35) to find exact solutions for the energy distributions associated with the aforementioned black hole models. 
On the Møller energy associated with black holes

1. Anti-de Sitter C-metric with spherical topology; In this black hole solution, the energy distribution is calculated as

$$
E(r)=\frac{m-\frac{q^{2}}{r}+\frac{1}{3}|\Lambda| r^{2}}{\sqrt{1-\frac{2 m}{r}+\frac{q^{2}}{r}+\frac{1}{3}|\Lambda| r^{2}}}
$$

and without cosmological constant the energy becomes the following form

$$
\lim _{\Lambda \rightarrow 0} E(r)=\frac{m-\frac{q^{2}}{r}}{\sqrt{1-\frac{2 m}{r}+\frac{q^{2}}{r}}}
$$

at a large distances

$$
\lim _{r \rightarrow \infty} E(r)=m \text {. }
$$

According to the Cooperstock hypothesis [37], the energy is confined to the region of non-vanishing energy-momentum tensor of matter and all non-gravitational fields.

2. Charged regular black hole; By using energy integrals and writing $\kappa=8 \pi$ in the second one defined in the teleparallel gravity, the energy is obtained both in general relativity and the teleparallel gravity as

$$
E(r)=m\left(1-\tanh \left(\frac{q^{2}}{2 m r}\right)\right)-\frac{q^{2}}{2 r}\left(1-\tanh ^{2}\left(\frac{q^{2}}{2 m r}\right)\right)
$$

at a large distances

$$
E(r)=m-\frac{q^{2}}{r^{2}}+\frac{q^{6}}{6 m^{2} r^{3}}-\frac{q^{10}}{40 m^{4} r^{5}}+O\left(\frac{1}{r^{6}}\right) .
$$

For the same black hole metric, by using the general relativity version of Møller energy, Radinschi [35] found that the energy is $E=m\left(1-\tanh \left(\frac{q^{2}}{2 m r}\right)\right)-\frac{q^{2}}{2 r}\left(1-\tanh ^{2}\left(\frac{q^{2}}{2 m r}\right)\right)$. Hence, we found that the teleparallel Møller's energy is the same as the general relativistic one. This energy is an acceptable one, because the black hole under consideration is charged and our result also sustains the viewpoint of Lassner [36] that the Møller energy-momentum complex is a powerful concept of energy and momentum. The energy distribution of a charged regular black hole reduces to $m$ as $r \rightarrow \infty$, corresponding to

$$
\lim _{r \rightarrow \infty} E(r)=m \text {. }
$$

3. Conformal scalar dyon black hole; The total energy distribution of a conformal scalar dyon black hole is found both in general relativity and the teleparallel gravity as

$$
E(r)=Q_{C S D}\left(1-\frac{Q_{C S D}}{r}\right) .
$$

In literature, Radinschi [35], the energy distribution of a conformal scalar dyon black hole was found $E=Q_{C S D}\left(1-\frac{Q_{C S D}}{r}\right)$. Our result is exactly the same as Radinschi's result. Hence, we found that (a) our result is an acceptable one, because the black hole under consideration is charged and (b) the general relativistic result and the tele-parallel gravitational one agree with each other. The energy distribution of a conformal scalar dyon black hole reduces to $Q_{C S D}$ as $r \rightarrow \infty$, corresponding to

$$
\lim _{r \rightarrow \infty} E(r)=Q_{C S D} .
$$


On the Møller energy associated with black holes

Hence, the energy(due to matter plus fields) is given by parameter $Q_{C S D}$ which is the charge for this metric.

4. Dyadosphere of a charged black hole; The energy of the dyadosphere of a charged black hole is obtained as

$$
E(r)=M-\frac{Q^{2}}{r}+\frac{\sigma Q^{4}}{5 r^{5}}
$$

In the previous paper Xulu [38], using the Einstein, Landau-Lifshitz, Papapetrou and Weinberg prescriptions found that the energy associated with the dyadosphere of a charged black hole is

$$
E_{E}=E_{L L}=E_{P}=E_{W}=M-\frac{Q^{2}}{2 r}+\frac{\sigma Q^{4}}{10 r^{5}} .
$$

It is obvious that in the dyadosphere region (where $r$ is small) the lats term of energy definition plays a very important role. As expected, $\sigma=0$ gives the energy distribution

$$
\begin{aligned}
& E_{E}=E_{L L}=E_{P}=E_{W}=M-\frac{Q^{2}}{2 r}, \\
& E_{M o l l e r}=M-\frac{Q^{2}}{r}
\end{aligned}
$$

which is for the Reissner-Nordström metric. At the large distances, our results and Xulu's one agree with each other.

$$
\lim _{r \rightarrow \infty} E(r)=M \text {. }
$$

5. Regular black hole; For this special case, we have the following energy

$$
E(r)=m r^{3}\left(r^{2}-2 e^{2}\right)\left(r^{2}+e^{2}\right)^{-5 / 2}
$$

which can be written as

$$
E(r)=m\left(1-\frac{9 e^{2}}{2 r^{2}}+\frac{75 e^{4}}{8 r^{4}}+O\left(\frac{1}{r^{6}}\right)\right)
$$

at a large distances $(r \rightarrow \infty)$

$$
\lim _{r \rightarrow \infty} E(r)=m \text {. }
$$

The energy is found the same as the one calculated using the general relativity version of the Møller definition by Sharif [39].

6. Charged topological black hole; We find the following energy

$$
E(r)=\frac{\frac{G m}{r}\left(1+\frac{G m}{r^{2}}\right)-\frac{\Lambda}{3} r^{3}}{\sqrt{-\frac{\Lambda r^{2}}{3}-\left(1+\frac{G m}{r^{2}}\right)^{2}}} .
$$

7. Charged massless black hole with a scalar Field;

$$
E(r)=-\left(-\frac{\Lambda r^{2}}{3}\right)^{-\frac{1}{2}} \frac{\Lambda}{3} r^{3} .
$$

8. The Schwarzschild-de Sitter space-time; We have the following energy distribution which depends on the mass of the Schwarzschild-de Sitter black hole.

$$
E(r)=M+\frac{r^{3}}{l^{2}} \text {. }
$$


In the special case where there is no cosmological constant, we obtain the energy distribution as

$$
\lim _{l \rightarrow \infty} E(r)=M
$$

\section{Summary and Discussions}

Møller showed that a tetrad description of a gravitational field equation allows a more satisfactory treatment of the energy-momentum complex than does general relativity. Therefore, we have also applied the super-potential method by Mikhail et. al. [9] to calculate the energy of the central gravitating body.

Recently, one of us [10] has considered the Møller energy-momentum definition in both general relativity and teleparallel gravity for the viscous Kasner-type metric and calculated the same energy. Also the result of that paper agrees with some of the previous papers by Cooperstock and Israelit, Rosen, Johri et al., Banerjee-Sen in general relativity and by Vargas in teleparallel gravity.

In this paper, in order to investigate the energy associated with a general black hole model with some of its special cases, we considered the Møller energy-momentum formulation in both general relativity and teleparallel gravity. We obtained that the energy distribution is the same in both of these different gravitation theories. It is also independent of the teleparallel dimensionless coupling constant, which means that it is valid not only in teleparallel equivalent of general relativity but also in any teleparallel model.

In the special cases, we obtained eight different black hole models, such as antide Sitter C-metric with spherical topology, charged regular black hole, conformal scalar dyon black hole, dyadosphere of a charged black hole, regular black hole, charged topological black hole, charged massless black hole with a scalar field, and the Schwarzschild-de Sitter space-time. For these black hole models, we obtained energy distribution (due to matter and fields including gravity) associated with them and also considered some limits of those results.

Finally, this paper sustains (a) the importance of the energy-momentum definitions in the evaluation of the energy distribution of a given spacetime and (b) the viewpoint of Lessner that the Møller energy-momentum complex is the powerful concept to calculate energy distribution in a given space-time.

\section{Acknowledgments}

We would like to thank Prof. K.S. Virbhadra and Dr. E.C. Vagenas for their suggestions and also The Turkish Scientific and Technical Research Council (Tübitak)-Feza Gürsey Institute, Istanbul, for the hospitality we received in summer terms 2002-2005. The work of MS was supported by Tübitak. 
On the Møller energy associated with black holes

\section{REFERENCES}

[1] A. Einstein, Sitzungsber. Preus. Akad. Wiss. Berlin (Math. Phys.) 778 (1915), Addendum-ibid. 799 (1915).

[2] R.C. Tolman, Relativity, Thermodynamics and Cosmology (Oxford Univ. Pres., London, P.227) (1934); A. Papapetrou, Proc. R. Irish. Acad. A52, 11 (1948); P.G. Bergmann and R. Thomson, Phys. Rev. 89, 400 (1953); C. Møller, Ann. Phys. (NY) 4, 347 (1958); Ann. Phys. (NY) 12, 118 (1961); L.D. Landau and E.M. Lifshitz, The Classical theory of fields (Pergamon Press, 4th Edition, Oxford) (1987); S. Weinberg, Gravitation and Cosmology: Principle and Applications of General Theory of Relativity (John Wiley and Sons, Inc., New York) (1972); A. Qadir and M. Sharif, Phys. Lett. A167, 331 (1992).

[3] G. Lessner, Gen. Relativ. Gravit. 28, 527 (1996).

[4] K.S. Virbhadra, Phys. Rev. D41, 1086 (1990); Phys. Rev. D42, 2919 (1990); Phys. Rev. D60, 104041 (1999); Pramana-J. Phys. 45, 215 (1995); N. Rosen and K.S. Virbhadra, Gen. Rel. Gravit. 25, 429 (1993); A. Chamorro and K.S. Virbhadra, Pramana-J. Phys. 45, 181 (1995); Int. J. Mod. Phys. D5, 251 (1996); J. M. Aguirregabiria, A. Chamorro and K.S. Virbhadra, Gen. Rel. Gravit. 28, 1393 (1996); S.S. Xulu, Int. J. Mod. Phys. A15, 4849-4856 (2000); E.C. Vagenas, Int. J. Mod. Phys. A18, 5781 (2003); Int. J. Mod. Phys. A18, 5949 (2003); Mod. Phys. Lett. A19, 213 (2004); Int. J. Mod. Phys. D14, 573 (2005); gr-qc/0602107.

[5] M.G. Albrow, Nature 241, 56 (1973).

[6] E.P. Tryon, Nature 246, 396 (1973).

[7] F. I. Cooperstock, Gen. Rel. Gravit. 26, 323 (1994); F. I. Cooperstock and M. Israelit, Found. Phys. 25, 631 (1995).

[8] N. Rosen, Gen. Rel. Gravit. 26, 319 (1994); V.B. Johri, D. Kalligas, G.P. Singh and C.W.F. Everitt, Gen. Rel. Grav. 27, 323 (1995); N. Banerjee and S. Sen, Pramana-J. Phys. 49, 609 (1997); S.S. Xulu, Int. J. Theor. Phys. 30, 1153-1161 (2000); M. Saltı, Nuovo Cimento B120, 53 (2005).

[9] T. Vargas, Gen. Rel. Gravit. 36, 1255 (2004); F.I. Mikhail, M.I. Wanas, A. Hindawi and E.I. Lashin, Int. J. Theor. Phys. 32, 1627 (1993); G.G.L. Nashed, Phys. Rev. D66, 064015 (2002).

[10] M. Saltı and A. Havare, Int. J. Mod. Phys. A20, 2169 (2005); O. Aydogdu and M. Saltı, Astrophys. Space Sci. 299, 227 (2005); O. Aydogdu, M. Saltı and M. Korunur, Acta Phys. Slov. 55, (2005) 537; M. Saltı and O. Aydogdu, gr-qc/0511030; M. Saltı, Astrophys. Space Sci. 299, 159 (2005); Mod. Phys. Lett. A20, 2175 (2005); Acta Phys. Slov. 55, (2005) 563; O. Aydogdu, gr-qc/0509047.

[11] J.A. Plebanski and M. Demianski, Annals of Physics (NY)98, (1976) 98.

[12] O.J.C Dias and J.P.S. Lemos, Phys. Rev. D67, (2003) 064001.

[13] E. Ayon-Beato and A. Garcia, Phys. Lett. B464, 25 (1999)

[14] K. Hayashi and T. Shirafuji, Phys. Rev. D19, 3524 (1978)

[15] K.S. Virbhadra and J.C. Parikh, Phys. Lett. B331, 302 (1994)

[16] R. Ruffini, Yamada Conference ob Black Holes and High Energy Astrophysics (Edited by H. Salto, Univ. Acad. Press., Tokyo, 1998)

[17] G. Preparata, R. Ruffini and S.-S. Wue, $A$ and A L87, 338 (1998)

[18] R. Ruffini, C. L. Bianco, S.-S. Xue, P. Chardonnet and F. Fraschetti, Int. J. Mod. Phys. D12, 173 (2003).

[19] V.A. De Lorenci, N.Figueredo, H.H. Fliche and M. Novello, Phys. Lett. B482, 134 (2000).

[20] J. Bardeen, Proc.GR5 (Tiflis, USSR, 1968).

[21] A. Borde, Phys. Rev. D50, 3692 (1994); Phys. Rev. D50, 7615 (1997)

[22] C. Martinez and J.P. Staforelli, hep-th/0512022.

[23] S. Shankaranarayanan, Phys. Rev. D67, 084026 (2003)

[24] R. Weitzenböck, Invariantten theorie (Gronningen: Noordhoff, 1923)

[25] K. Hayashi and T. Shirafuji, Phys. Rev. D19, (1978) 3524.

[26] V.V. de Andrade and J.G. Pereira, Phys. Rev. D56, (1997) 4689.

[27] C. Møller, Mat. Fys. Medd. K. Vidensk. Selsk. 39, (1978) 13; Mat. Fys. Medd. K. Vidensk. Selsk. 
1, (1961) 10.

[28] D. Saez, Phys. Rev. D27, (1983) 2839.

[29] H. Meyer, Gen. Rel. Gravit. 14, (1982) 531.

[30] K. Hayashi and T. Shirafuji, Prog. Theor. Phys. 64, (1980) 866; 65, (1980) 525.

[31] F.W. Hehl, J. Nitsch and P. von der Heyde: in General Relativity and Gravitation, edited by A. Held (Plenum, New York) (1980), p.329.

[32] H.P. Robertson, Ann. Math. (Princeton) 33, (1932) 496.

[33] Wolfram Research, Mathematica 5.0 (2003).

[34] TCI Software Research, Scientific Workplace 3.0 (1998).

[35] I. Radinschi, Mod. Phys. Lett. A15, 2171 (2000); Mod. Phys. Lett. A20, 673 (2001); I-Ching Yang and I. Radinschi, Chin. J. Phys. 41, 326 (2003).

[36] G. Lessner, Gen. Relativ. Gravit. 28, 527 (1990)

[37] F.I. Cooperstock and S.A. Richardson, in Proc. 4th Canadian Conf. on General Relativity and Relativistic Astrophysics, held 16-18 May, 1991 at University of Winnipeg. Edited by G. Kunstatter, D.E. Vincent and J.G. Williams, World Scientific, Singapore, 1992, p.110.

[38] S.S. Xulu, preprint gr-qc/0304081.

[39] M. Sharif; Nuovo Cim. B19 (2004) 463. 